\title{
The Measurement of the Mass of the W Boson from the Tevatron
}

\author{
Randy Thurman-Keup ${ }^{1}$ \\ Argonne National Lab / HEP-362 \\ 9700 South Cass Avenue \\ Argonne, IL 60439
}

\begin{abstract}
This paper presents measurements of the mass of the $W$ vector boson from the CDF and D $\emptyset$ experiments using data collected at $\sqrt{s}=1.8 \mathrm{TeV}$ during the 19941995 data taking run. CDF finds a preliminary mass of $M_{W}=80.43 \pm 0.16 \mathrm{GeV}$ and D $\emptyset$ measures a mass of $M_{W}=80.44 \pm 0.12 \mathrm{GeV}$.
\end{abstract}

\section{INTRODUCTION}

During the 1994-1995 collider run of the Fermilab Tevatron, the CDF and D $\varnothing$ experiments collected data corresponding to integrated luminosities of $90 \mathrm{pb}^{-1}$ and $82 \mathrm{pb}^{-1}$ respectively. From this data, CDF extracted $\sim 21,000 W \rightarrow \mu \nu$ events while $\mathrm{D} \varnothing$ obtained $\sim 28,000 W \rightarrow e \nu$ events. These $W$ events were used to make measurements ${ }^{2}$ of the mass of the $W$ boson [1].

\section{A Motivation}

When going from tree level in the Standard Model (SM) to next-to-leading order, the relation between the mass of the $W$ boson and the other SM parameters gets modified as follows:

$$
\Rightarrow \quad M_{W}^{2}=\left(\frac{\pi \alpha_{E M}(0)}{\sqrt{2} G_{F} \sin ^{2} \theta_{W}}\right) \rightarrow\left(\frac{\pi \alpha_{E M}\left(M_{Z}^{2}\right)}{\sqrt{2} G_{F} \sin ^{2} \theta_{W}}\right) \frac{1}{1-\Delta r}
$$

where $\Delta r$ embodies the loop corrections to the $W$ propogator [2]. The dominant contributions to the corrections come from the top quark which introduces a quadratic dependence on its mass, and the higgs boson which produces a logarithmic dependence on its mass. These dependencies allow one to probe for the

1) representing the $C D F$ and $D \emptyset$ collaborations

2) The CDF measurement is only a preliminary measurement.

* Work supported in part by the U.S. Department of Energy, Division of High Energy Physics, Contract W-31-109-ENG-38.
The submitted manuscript has been created by the University of Chicago as Operator of Argonne National Laboratory ("Argonne") under Contract No. W-31-109-ENG-38 with the U.S. Department of Energy. The U.S. Government retains for itself, and others acting on its behalf, a paid-up, nonexclusive, irrevocable worldwide license in said article irrevocable worldwide license in said article tribute copies to the public, and perform publicly and display publicly, by or on behalf of the Government. 


\section{DISCLAIMER}

This report was prepared as an account of work sponsored by an agency of the United States Government. Neither the United States Government nor any agency thereof, nor any of their employees, make any warranty, express or implied, or assumes any legal liability or responsibility for the accuracy, completeness, or usefulness of any information, apparatus, product, or process disclosed, or represents that its use would not infringe privately owned rights. Reference herein to any specific commercial product, process, or service by trade name, trademark, manufacturer, or otherwise does not necessarily constitute or imply its endorsement, recommendation, or favoring by the United States Government or any agency thereof. The views and opinions of authors expressed herein do not necessarily state or reflect those of the United States Government or any agency thereof. 


\section{DISCLAIMER}

Portions of this document may be illegible in electronic image products. Images are produced from the best available original document. 
higgs given a precision measurement of the $W$ mass. In addition, Standard Model extensions and/or replacements produce their own corrections and here again, a precision measurement may be used to uncover these theories.

\section{B Tevatron Environment}

The majority of $W$ events at the Tevatron are produced from s channel quark- antiquark interactions. The $W$ 's of interest in this measurement are the ones that decay to muon-neutrino or electron-neutrino pairs, since these are the cleanest decays. Unfortunately, the fact that the neutrino is undetected, prevents us from measuring the invariant mass of the $W$. One could infer the neutrino 3-momentum by requiring momentum conservation in the event if it weren't for the fact that the energy of the incident quarks is not known. Unlike electron-positron colliders, the quarks are bound inside the (anti)protons and their momentum is governed by parton distribution functions (PDFs). Fortunately we can still enforce momentum conservation in the plane transverse to the beam direction since the since the incident quarks have essentially no transverse momentum.

This leaves us with two possible quantities from which to extract the mass: the transverse momentum of the lepton, $p_{T}^{\ell}$, or the transverse mass of the leptonneutrino pair, $M_{T}=\sqrt{2 p_{T}^{e} p_{T}^{\nu}(1-\cos \Delta \phi)}$. The transverse mass is analogous to the invariant mass except that only components of momentum transverse to the beam are used. The transverse mass is less sensitive than $p_{T}^{\ell}$ to the transverse momentum of the $W^{3}$ and is upwardly bounded by $M_{W}$ thereby still providing sensitivity to the mass. It is, however, more sensitive to the energy resolution of the calorimeter (see Sec. III A) since it depends on the inferred momentum of the neutrino. Which method is ultimately chosen depends on the relative precision of the measurement of the transverse momentum distribution of the $W$ (increased systematic uncertainty in $p_{T}$ ) when compared to the calorimeter resolution (increased statistical uncertainty in $M_{T}$ ). In the present case, $M_{T}$ wins.

\section{Event Selection}

The signature for $W$ events is a high momentum muon or electron $\left(p_{T}>\right.$ $25 \mathrm{GeV})^{4}$, and large missing energy from the undetected neutrino ( $\not t_{t}>25 \mathrm{GeV}$ ). This missing energy is the transverse momentum needed to balance the visible transverse momentum in the event. The visible momentum is the sum of the muon or electron momentum and a vector sum of the energy in the calorimeter towers of the detector (not including the energy from the lepton of course; see Sec. III A). The lepton must be central $(|\eta|<1)$ and satisfy various quality cuts. For D $\varnothing$, these

3) Corrections are of $\mathcal{O}\left(\beta^{2}\right)$ compared with $\mathcal{O}(\beta)$ for $p_{T}^{\ell}$.

4) Throughout this paper, $\hbar=c=1$; thus mass, momentum, and energy all have units of $\mathrm{eV}$. 
cuts require the electron to be isolated, to have a calorimeter shower shape consistent with Monte Carlo expectations, and to have a track pointing at the calorimeter cluster. For CDF, the muon is required to have deposited energy in the calorimeter consistent with a minimum ionizing particle, to have a track pointing at the hits in the muon chambers, and to not be consistent with a cosmic ray. There is also a requirement that the vector sum of the energy in the calorimeter (not including the contribution from the lepton), $\vec{u}$, be less than $15 \mathrm{GeV}$ for $\mathrm{D} \varnothing$ and less than $20 \mathrm{GeV}$ for CDF. This serves to further reduce the background from QCD inspired - processes and results in cleaner events. After these requirements are placed on the data sample, CDF has 21,000 W's remaining and D $\varnothing$ has 28,000 W's left.

There are other datasets that are used in this analysis, chief among them being $Z \rightarrow \ell^{+} \ell^{-}$. The requirements on $Z$ events are similar to $W$ 's for one lepton and typically loosened somewhat for the other lepton resulting in $2200 Z$ 's for $D \varnothing$ and $1400 Z$ 's for CDF.

\section{LEPTON MOMENTUM CALIBRATION}

CDF and DØ take similar approaches to calibrating the energy scale for the lepton. Both involve comparing measured mass resonances with known mass values. In the case of CDF, $J / \psi \rightarrow \mu \mu$ decays are used to set the momentum scale of the tracking chamber; at $\mathrm{D} \varnothing, Z \rightarrow e e, \pi^{0} \rightarrow \gamma \gamma$, and $J / \psi \rightarrow e e$ are used to set the energy scale of the calorimeter.

\section{A CDF Momentum Scale}

The momentum scale of the tracking chamber / magnetic field is set using the invariant mass distribution of $\sim 250,000 \mathrm{~J} / \psi \rightarrow \mu \mu$ events (Fig. 1), which is fit using a simulated lineshape. The data are corrected for magnetic field variations over the course of data taking and the momenta are corrected for energy lost in the material before the tracking chamber. The $J / \psi$ simulation includes QED radiative contributions and both prompt and $B$ decay sources of $J / \psi$ 's. These two effects can be seen in Figure 1. The latter is important because the tracks from the muons are constrained to originate from the beamline which introduces a systematic bias in tracks that did not originate from the beamline. This beam constraint is applied to $J / \psi$ events to uncover any possible unknown biases that may affect the $W$ mass measurement.

Fitting the lineshape to the $J / \psi$ data results in a mass of $3096.2 \pm 1.5 \mathrm{MeV}$. This translates to a momentum scale of $0.99977 \pm 0.00048$ leading to an uncertainty of $40 \mathrm{MeV}$ on the $W$ mass. The dominant uncertainties in the momentum scale are the $\mathrm{dE} / \mathrm{dx}$ energy loss correction and the extrapolation of the momentum scale from the $J / \psi$ mass to the $W$ mass.

Muon Energy Loss - The muons from $J / \psi$ decays traverse material before entering the tracking volume and thus lose energy. The amount of energy they lose 

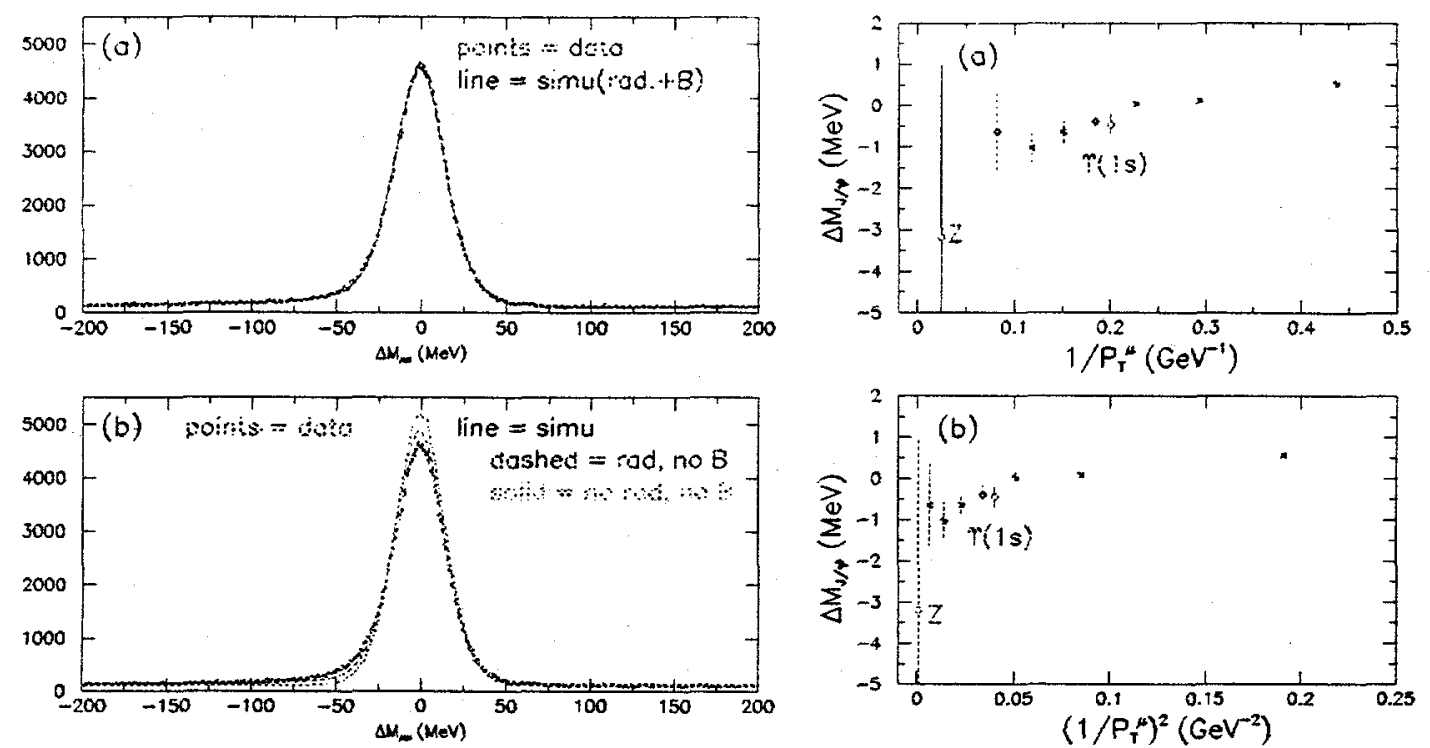

FIGURE 1. Left: a) Invariant mass distribution of $J / \psi \rightarrow \mu \mu$ events. b) The effect of adding various features to the simulation. Right: $J / \psi$ mass as a function of a) inverse $p_{T}^{\mu}$ and $b$ ) inverse $\left(p_{T}^{\mu}\right)^{2}$.

depends on the amount and type of material. The amount of material can be obtained using photon conversions to electron-positron pairs. These conversions are used to map out the material in the inner detector, and when combined with knowledge about the composition of the various structures provide the necessary corrections. The uncertainty of $1.0 \mathrm{MeV}$ produced in the $J / \psi$ mass is due to uncertainties in the material types in the various regions and to residual variation in the $J / \psi$ mass with region.

Extrapolation to the $W$ - The momentum scale is obtained using muons from $J / \psi$ decays which have an average momentum of $4 \mathrm{GeV}$. This is a long way from the typical $38 \mathrm{GeV}$ momenta of $W$ decay muons. Fortunately the relevant quantity is not momentum but inverse momentum, which is proportional to the curvature of the track. This is what the tracking chamber measures and where deviations from expected behavior should occur. Figure 1 shows the variation of $J / \psi$ mass with inverse momenta. The advantage is that the distance in inverse momenta from the $J / \psi$ to the $W$ is shorter than the spread in the $J / \psi$ data making for an effortless extrapolation. Since the extrapolated difference is small and since wrong $\mathrm{dE} / \mathrm{dx}$ corrections, for example, can fake a variation here, it is taken as an uncertainty rather than a correction. This uncertainty, if expressed in terms of the $J / \psi$ mass, is also $1.0 \mathrm{MeV}$. 

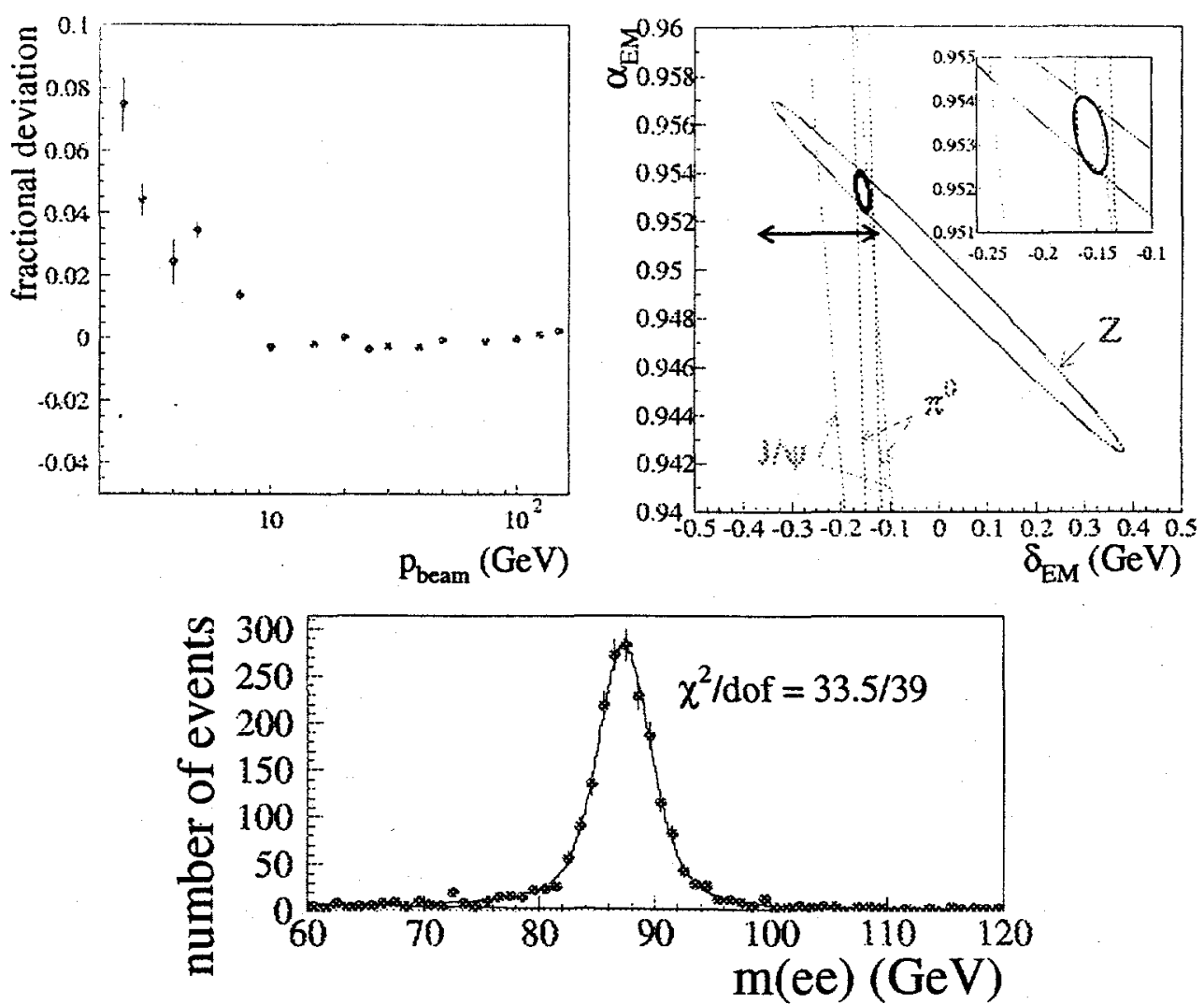

FIGURE 2. Top Left: Deviations from the expected response for the DØ electromagnetic calorimeter for electrons as a function of the electron energy. Top Right: The contours in the scale parameter's plane for $J / \psi \rightarrow e e, \pi^{0} \rightarrow \gamma \gamma$, and $Z \rightarrow e e$. The thick contour is all three combined including only the statistical component. The double arrow indicates the systematic uncertainty in $\delta$ due to deviations in the testbeam results at low energy (top left plot). Bottom: Invariant mass distribution of electron pairs near the $Z$ compared with simulation where the simulation is adjusted for the energy scale to allow comparison with data.

\section{B D $\varnothing$ Energy Scale}

The $D \varnothing$ calorimeter is a Uranium/Liquid Argon sampling calorimeter which, since the LAr has unit gain, is extremely stable over time. This enables electron testbeam data to be used to obtain a simple functional form for the energy scale (Fig. 2). The testbeam results indicate that the calorimeter response is very linear and only deviates from the expected response below $10 \mathrm{GeV}$. Thus a linear function is used to relate the true energy to the measured energy, $E_{\text {meas }}=\alpha_{E M} E_{\text {true }}+\delta_{E M}$. The deviations from this linear behavior are treated as systematic uncertainties.

Like CDF, D $\varnothing$ determines the constants in the above equation by comparing mass resonances with known masses. Three resonances are used: $J / \psi \rightarrow e e, \pi^{0} \rightarrow \gamma \gamma$, and $Z \rightarrow e e$.

$J / \psi \rightarrow$ ee Decays - The relation between the measured $J / \psi$ mass peak and the 

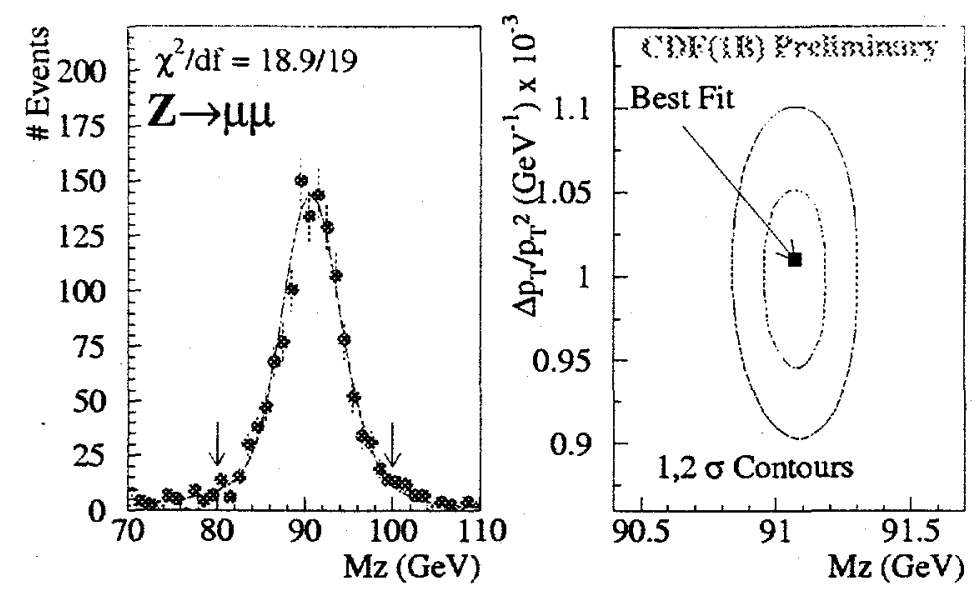

FIGURE 3. Left: Invariant mass distribution of $Z \rightarrow \mu \mu$ events from CDF. The width of the peak is a convolution of the linewidth of the $Z$ and the momentum resolution. Right: Contours in momentum resolution and mass with best fit point.

$J / \psi$ mass is determined from simulation to be $m(e e)=\alpha_{E M} m_{J / \psi}+0.56 \delta_{E M}$. This equation defines a contour in the $\alpha_{B M} \delta_{E M}$ plane (Fig. 2).

$\pi^{0} \rightarrow \gamma \gamma$ Decays - The decay $\pi^{0} \rightarrow \gamma \gamma$ is detected by identifying the electrons resulting from the conversions of the two photons ${ }^{5}$. Because the calorimeter clusters from the 4 electrons overlap, a quantity called the symmetric mass is constructed which is equal to the invariant mass if the energy of the two photons is equal. As with the $J / \psi \rightarrow e e$ decays, a similar equation relating the symmetric mass to the actual mass can be obtained and again results in a contour in the $\alpha_{E M}-\delta_{E M}$ plane (Fig. 2).

$\boldsymbol{Z} \rightarrow$ ee Decays - Both the previous resonances are low masses and as such do a good job of constraining $\delta_{E M}$ but do a poor job of constraining $\alpha_{E M}$. To constrain $\alpha_{B M}$ the $Z \rightarrow e e$ resonance is used. The $Z$ has the added benefit that the decay electrons are not monochromatic thus providing another albeit weak measurement of $\delta_{E M}$. Again, a contour in $\alpha_{E M} \delta_{E M}$ is found and together with the $J / \psi$ and $\pi^{0}$ provides a tight constraint on $\alpha_{B M}$ and $\delta_{E M}$ (Fig. 2). The best fit returns $\delta_{E M}=-0.16_{-0.21}^{+0.03} \mathrm{GeV}$ and $\alpha_{E M}=0.9533 \pm 0.0008$ where the uncertainties include systematic contributions.

\section{- C Energy and Momentum Resolutions}

The lepton energy or momentum resolution is obtained by fitting for the width of either the $Z \rightarrow e e$ (DØ; Fig. 2) or $Z \rightarrow \mu \mu$ (CDF; Fig. 3) distributions.

The electron energy resolution for $\mathrm{D} \emptyset$ is parameterized as

$$
\frac{\sigma_{E}}{E}=\frac{13.5 \%}{\sqrt{E \sin \theta}} \oplus \kappa \oplus \frac{n}{E}
$$

5) Each of the two conversion pairs appears as a doubly ionized track in the tracking chamber 
where the first term (stochastic) is determined from testbeam data and the last term $(n / E)$ is the contribution from other energy in the event and is determined from calorimeter towers near the electron. The constant term, $\kappa$, is measured from the width of the $Z$ and is found to be $\left(1.15_{-0.36}^{+0.27}\right) \%$.

The momentum resolution for CDF is parameterized as $\sigma_{1 / p_{T}}=\kappa \cdot\left(1 / p_{T}\right)$ since the tracking chamber measures the curvature of a track which is proportional to $1 / p_{T}$. The constant is extracted from the width of the $Z$ and is $(0.101 \pm 0.005) \%$.

\section{RECOIL MEASUREMENT AND CALIBRATION}

\section{A Recoil Measurement}

There are only two quantities to measure in every $W$ event: the lepton $p_{T}$, and the transverse recoil momentum, $\vec{u}$, from the $p_{T}$ of the $W$. Together they can be used to infer the neutrino $p_{T}$. The recoil momentum is defined as $\vec{u}=\sum_{i}\left(E_{i} \cdot \sin \theta_{i}\right) \hat{\phi}_{i}$ where the sum runs over calorimeter towers and $\theta$ and $\hat{\phi}_{i}$ are the polar angle and azimuthal unit vector of the tower containing energy $E$. The towers containing energy deposited by the lepton are removed from the sum; however, the removed towers also contain small contributions from the recoil which must be accounted for. The CDF measurement replaces the removed towers with an average recoil event energy determined from nearby towers. The $D \emptyset$ measurement on the other hand duplicates the removal in the simulated data and also corrects the simulated electron energy to account for this small recoil contamination.

\section{B Recoil Calibration}

The calibration of the recoil measurement is obtained from $Z$ data where the recoil measurement can be compared to the $p_{T}$ of the $Z$ measured with the leptons. There is a slight complication in that $\vec{u}$ contains not only the recoil energy but also the energy from the (anti)proton breakup plus any overlapping $\bar{p} p$ interaction. Thus the calibration also includes a minimum bias component.

D $\varnothing$ Calibration - The parameterization of $\vec{u}$ is given by

$$
\begin{aligned}
& =\quad \vec{u}=\left[R_{\text {rec }} \vec{q}_{T}+s_{r e c} \sqrt{R_{\text {rec }} q_{T}} \hat{q}_{t}\right]-\Delta \vec{u}+\alpha_{m b} \vec{E}_{T}^{m b} \\
& R_{\text {rec }}=\alpha_{\text {rec }}+\beta_{\text {rec }} \log \left(q_{T}\right)
\end{aligned}
$$

where $R_{\text {rec }}$ represents the recoil response, $s_{\text {rec }}$ is the response resolution, $\Delta u$ is the small correction for the tower removals mentioned in Section III A, and the last term is a literal minimum bias event weighted by $\alpha_{m b}$.

The form of $R_{r e c}$ is obtained from a Herwig-Geant $Z \rightarrow e e$ simulation (Fig. 4) and does a good job of describing the $D \varnothing$ jet response. $Z$ data is used to constrain the parameters $\alpha_{r e c}$ and $\beta_{r e c}$ and the values obtained $\left(\alpha_{r e c}=0.693 \pm 0.060\right.$ and 

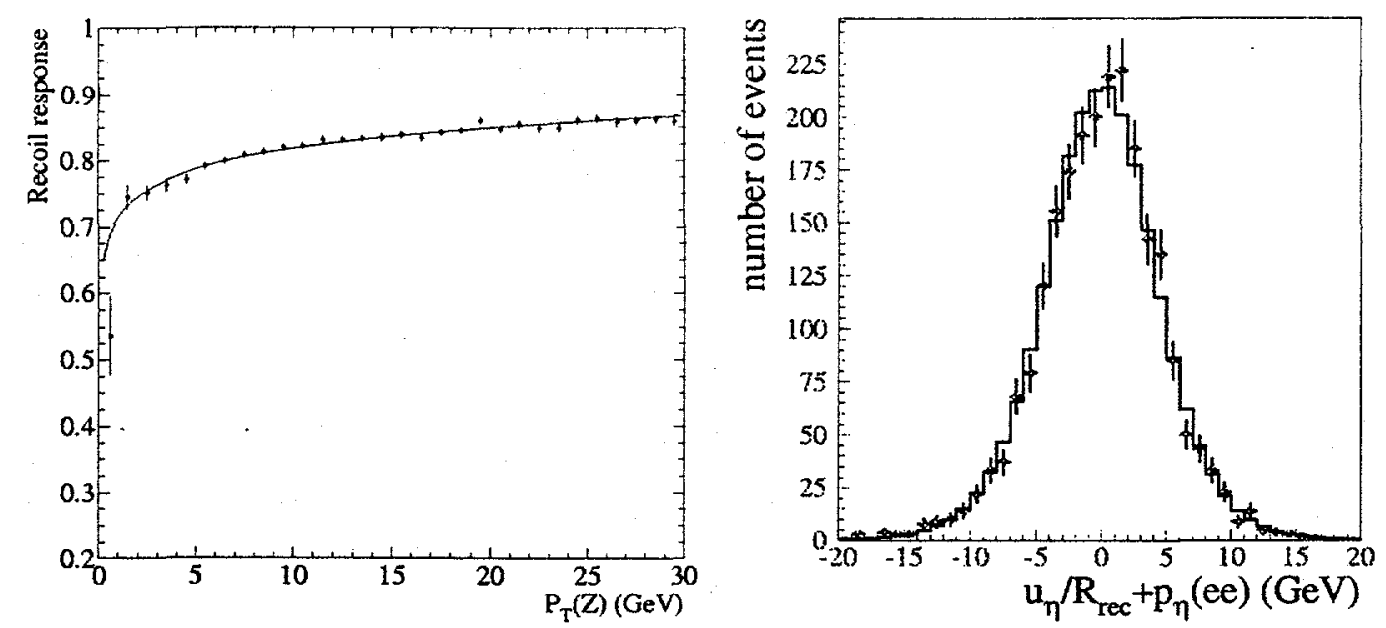

FIGURE 4. Left: Simulated detector response to recoil from $Z$ 's in Herwig-Geant $Z \rightarrow e e$ simulation. Right: Difference between $p_{\eta}$ and $u_{\eta}$ after being corrected by the response function. The points are the data and the histogram is the simulation. This distribution should have a mean of zero and a width characteristic of the recoil resolution. ( $\eta$ is the angular bisector of the two electrons and is the axis with the least sensitivity to the electron energy resolution.)

$\left.\beta_{r e c}=0.040 \pm 0.021\right)$ agree with the Herwig-Geant values of 0.713 and 0.046 . The parameter $s_{\text {rec }}$ is the recoil part of the resolution parameterization and is also obtained from $Z \rightarrow e e$ events along with the parameter $\alpha_{m b}$ representing the nonrecoil part of the resolution. This non-recoil part is modeled by a minimum bias event (chosen such that the luminosity distribution is the same as in the $Z$ data) multiplied by a weight, $\alpha_{m b}$, which is constrained by the $Z$ data $^{6}$. Comparisons of Monte Carlo $Z \rightarrow e e$ events with data in Figure 4 show good agreement in both the mean of the distribution (response) and the width (resolution).

CDF Calibration - The CDF parameterization of $\vec{u}$ is

$$
\begin{gathered}
\vec{u}=R_{r e c} \vec{q}_{T}+S \vec{\sigma}_{m b} \\
R_{r e c}=\alpha_{r e c}+\beta_{r e c} e^{-\lambda q_{T}}
\end{gathered}
$$

where, as with $\mathrm{D} \emptyset, R_{\text {rec }}$ is the recoil response and, unlike $\mathrm{D} \emptyset$, all the resolution is contained in the second term.

The form of $R_{\text {rec }}$ is obtained from $Z$ data and is plotted in Figure 5. The parameters are additionally constrained by $W$ data distributions to improve the uncertainty in the recoil response.

The resolution term handles both resolution from the minimum bias contribution and from the recoil response resolution. This works because for most $W$ 's, the recoil tends to look like the minimum bias contribution and thus one resolution is a fairly good approximation. The starting point for the resolution term is minimum bias data in which energy fluctuations are parameterized in terms of total energy in the

6) $\alpha_{m b}$ must be adjusted for $W$ data which have a different luminosity distribution. 

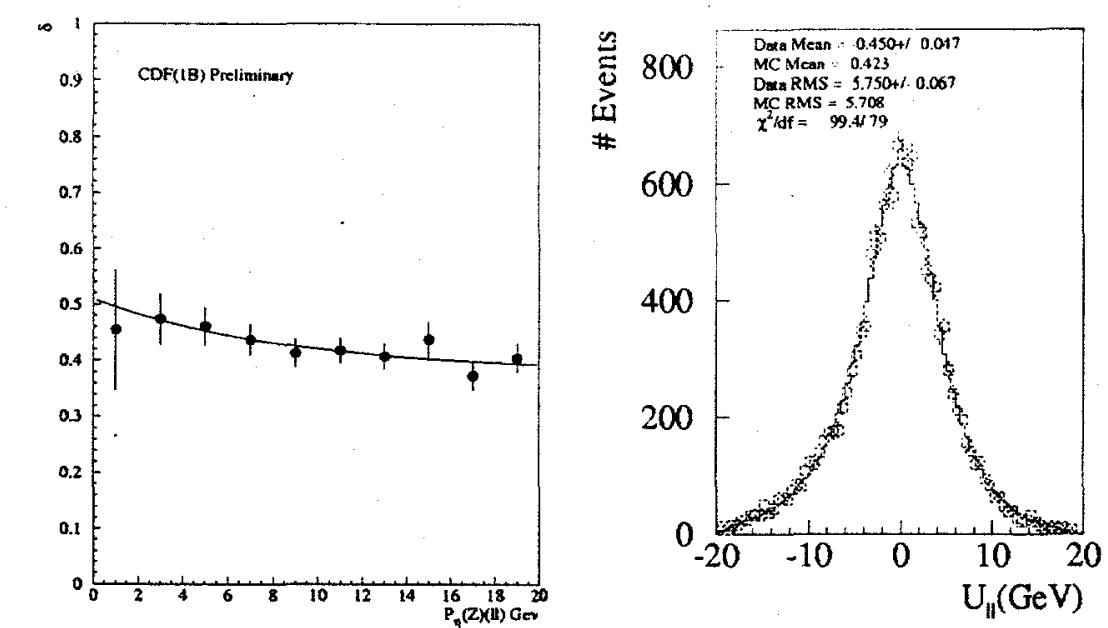

FIGURE 5. Left: Plot of $1-R_{r e c}$ vs. $p_{\eta}$ for $Z$ events. The points are both $Z \rightarrow e e$ and $Z \rightarrow \mu \mu$ events and the solid line is the fit to these points. Right: Comparison of $u_{\|}$from $W$ data and simulation. The variable $u_{\|}$is the projection of $\vec{u}$ onto the lepton momentum axis. This quantity should have a negative mean since the lepton is boosted in the direction of the $W$ (opposite $\vec{u}$ ).

event which itself is a function of the luminosity. This is weighted by $S$ which is constrained from $|\vec{u}|$ distributions in the $W$ data.

After obtaining the parameters for the recoil model, one can compare the simulation with $W$ data. Figure 5 is one such comparison of $u_{\|}$in data and simulation from CDF. Comparisons from $D \varnothing$ show similar agreement.

\section{MONTE CARLO SIMULATION}

The simulations used by both $\mathrm{CDF}$ and $\mathrm{D} \varnothing$ are similar in form. They start with a tree level calculation including parton distribution functions (PDF's). In an effort to separate out the various effects of the PDF's, D $\varnothing$ parameterizes the $Q^{2}$ effect of the PDF's as $e^{-\beta Q} / Q$ where $\beta$ is determined from Monte Carlo studies. The choice of which PDF to use is somewhat debatable. CDF attempts to constrain the allowed range of PDF's using $W$ decay charge asymmetry data where the asymmetry is a function of the $\mathrm{u}$ to $\mathrm{d}$ ratio. The $W$ lineshape is also dependent on this u-d ratio and thus the asymmetry can be used to set limits on the allowed PDF's. Unfortunately, the range of current PDF's does not fill the allowed asymmetry space making it difficult to use this method. $D \emptyset$ has chosen a small set of recent PDF's and taken the variation as a systematic uncertainty.

NLO QCD contributions are incorporated using a calculation [3] which matches the $\mathcal{O}\left(\alpha_{s}^{2}\right)$ large $q_{T}$ perturbative result with a small $q_{T}$ soft gluon resummation. There are 3 parameters $(g 1, g 2, g 3)$ plus $\Lambda_{Q C D}$ in the most recent incarnation of this calculation. CDF fixes $g 3$ to the Ladinsky-Yuan value and constrains $g 1$ and $g 2$ with $Z$ data ( $\Lambda_{Q C D}$ is varied by varying PDF's). DØ sets $g 1$ and $g 3$ to the 

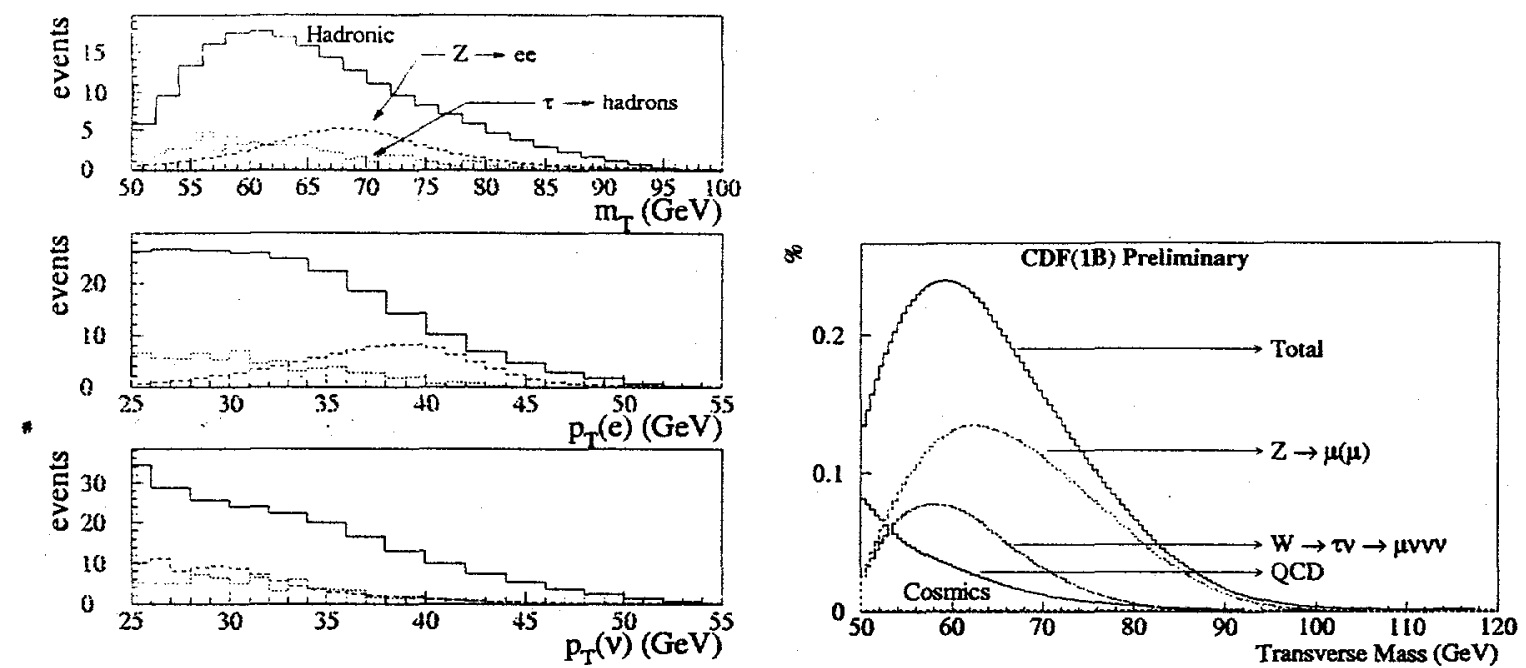

FIGURE 6. Left: D $\emptyset$ background shapes for transverse mass and lepton and neutrino $p_{T}$ distributions. The hadronic background is $(1.3 \pm 0.2) \%$, the $Z$ background is $(0.42 \pm 0.08) \%$, and the $\tau \rightarrow$ hadrons is $0.24 \%$. There is also a $W \rightarrow \tau \nu \rightarrow e \nu \nu \nu$ which is generated simultaneously with the $W \rightarrow e \nu$ events and contributes $1.6 \%$ events. Right: CDF background shapes for the transverse mass distribution. The dominant background is $Z \rightarrow \mu \mu$ where one muon is not detected in the tracking chamber and amounts to (3.6 \pm 0.5$) \%$. The other backgrounds are $W \rightarrow \tau \nu \rightarrow \mu \nu \nu \nu$ at $0.8 \%, Q C D$ at $(0.4 \pm 0.2) \%$, and Cosmic Rays which add $(0.1 \pm 0.05) \%$.

Ladinsky-Yuan values and constrains $g 2$ with $Z$ data while allowing $\Lambda_{Q C D}$ to vary within reasonable bounds.

The decay model includes QED radiative effects using a calculation by Berends and Kleiss [4]. The results were checked by both CDF and D $\varnothing$ using two-photon generators [5].

Backgrounds are included in the simulated lineshape and are detailed in Figure 6.

\section{RESULTS}

The mass of the $W$ is extracted using a likelihood fit of the Monte Carlo lineshapes to the data. The most precise value is obtained from fits to the transverse mass (Fig. 7).

The results for CDF and $D \emptyset$ are

$$
\begin{gathered}
M_{W}^{D \varnothing}=80.44 \pm 0.12 \mathrm{GeV} \\
M_{W}^{C D F}=80.43 \pm 0.16 \mathrm{GeV} .
\end{gathered}
$$

The uncertainties in these measurements are documented in Table 1. Combining these numbers with previous measurements by CDF and D $\varnothing$ and with measurements made by the LEP II experiments and $\mathrm{NuTeV}$ leads to a world average of 

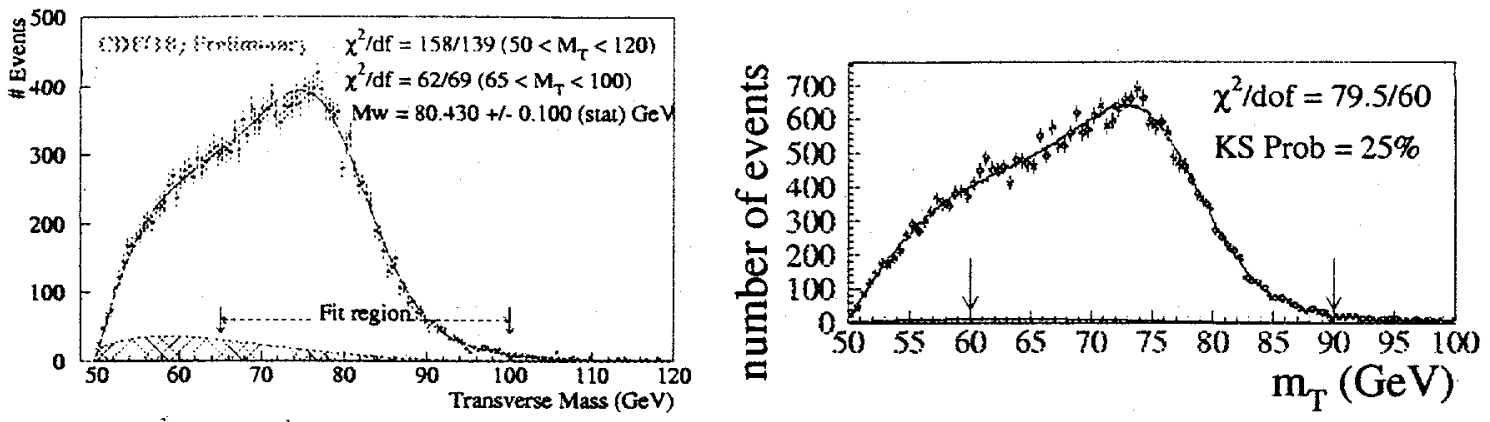

FIGURE 7. Left: CDF transverse mass distribution compared with simulation. The fit region is 65 to $100 \mathrm{GeV}$. Right: Same plot for $\mathrm{D} \emptyset$ with a fit region of 60 to $90 \mathrm{GeV}$.

\begin{tabular}{|c|c|c|c|}
\hline Uncertainty & $\begin{array}{r}\mathrm{CDF} \\
(\mathrm{M}\end{array}$ & $\begin{array}{l}\mathrm{D} \emptyset \\
\mathrm{V})\end{array}$ & \\
\hline Statistical & 100 & 70 & \\
\hline Momentum / Energy Scale & 40 & 65 & 95 (stat) \\
\hline Calorimeter Linearity & - & 20 & \\
\hline Lepton Resolution & 25 & 20 & \\
\hline Recoil Modeling & 90 & 40 & \\
\hline Input $W p_{T}$ and PDF's & 50 & 25 & \\
\hline Radiative Decays & 20 & 15 & \\
\hline Higher Order Corrections & 20 & - & \\
\hline Backgrounds & 25 & 10 & \\
\hline Lepton Angle Calibration & - & 30 & \\
\hline Fitting & 10 & - & \\
\hline Miscellaneous & 20 & 15 & \\
\hline Systematics Total & 115 & 70 & \\
\hline Total (MeV) & 155 & 120 & \\
\hline
\end{tabular}

TABLE 1. Uncertainties in the $W$ mass measurement for both CDF and DØ. It is suspected that the $90 \mathrm{MeV}$ recoil modeling uncertainty for CDF is due in part to deficiencies in the model and should decrease in the final analysis. The energy scale uncertainty for $D \emptyset$ is dominated by the statistics of the $Z$ data.

$$
M_{W}=80.420 \pm 0.055 \mathrm{GeV} .
$$

These measurements are listed in Figure 8 together with the current best indirect determination of $M_{W}$. Also in Figure 8 is a graphical representation of the state of the art in precision electroweak testing. The data point is the world average from above.

\section{A The Future}

CDF is currently working on adding the electron decay channel to the mass measurement while improving the muon result. The expectations are that the 

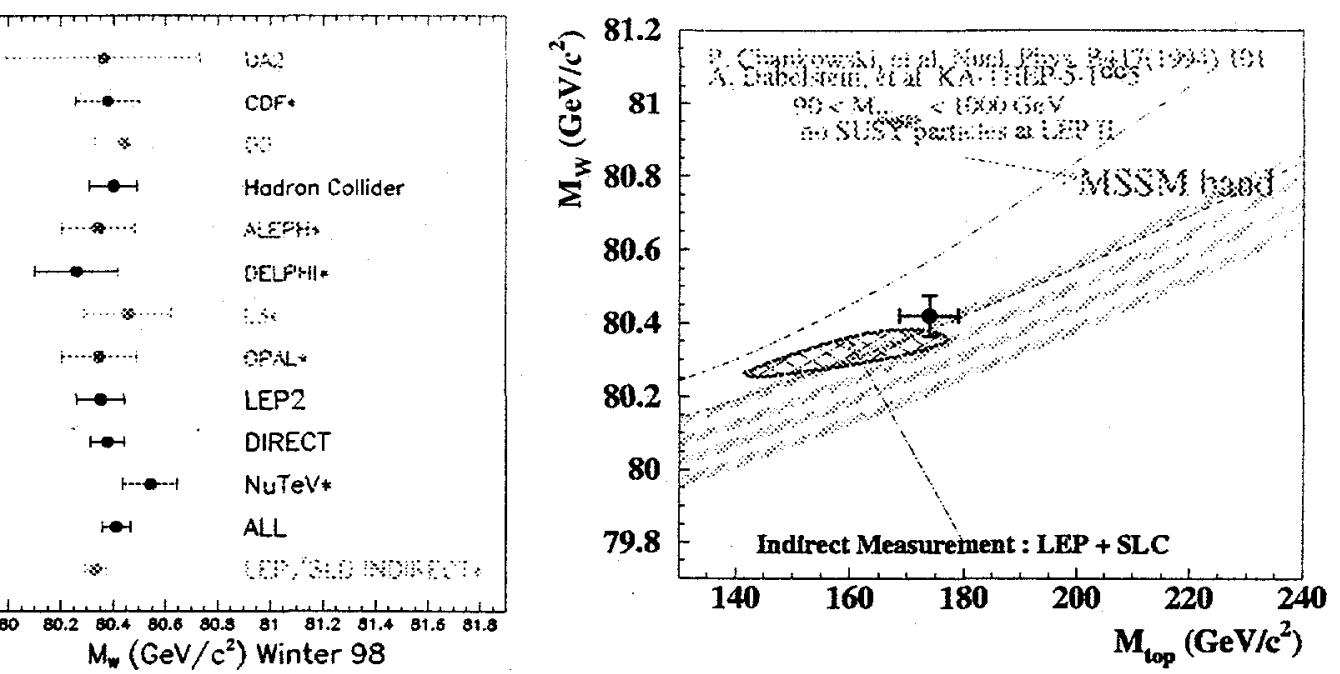

FIGURE 8. Left: $W$ mass measurements from around the world. The CDF and $D \emptyset$ points are combined numbers from all their respective data taking runs. Right: $W$ mass versus top mass for various higgs masses and SUSY.

CDF uncertainty can be reduced to a little under $100 \mathrm{MeV}$. D $\emptyset$ is working on increasing the pseudorapidity range of accepted electrons by including those that traverse the endcap calorimeter. Here too, the aim is to reach $100 \mathrm{MeV}$.

Both experiments are also upgrading the detectors in preparation for Run 2 of the Tevatron which will bring a factor of 20 more data. With this larger dataset and a lot of perseverence on the part of the experimenters, the uncertainty is expected to reach $\sim 40 \mathrm{MeV}$. Even farther in the future, TeV33 is supposed to supply another factor of 10 in data and a corresponding decrease in the $W$ mass uncertainty, perhaps as low as $20-30 \mathrm{MeV}$.

\section{REFERENCES}

1. B. Abbott et al., (D $\varnothing$ Collaboration), Phys. Rev. Lett. 80, 3008 (1998), and submitted to Phys. Rev. D. More information on both the CDF and D $\varnothing$ measurements can be found at http://www-cdf.fnal.gov/physics/ewk/ewk.html and http://ww-do.fnal.gov/public/wz/ewk public.html.

2. W. Hollik and W. Marciano, Precision Tests of the Standard Electroweak Model, ed. by P. Langacker (World Scientific, Singapore, 1994).

3. G.A. Ladinsky and C.P. Yuan, Phys. Rev. D 50, 4239 (1994); P.B. Arnold and R.P. Kauffman, Nucl. Phys. B 349, 381 (1991).

4. F.A. Berends and R. Kleiss, Z. Phys. C 27, 365 (1985); F.A. Berends et al., Z. Phys. C 27, 155 (1985).

5. CDF used PHoToS and D $\varnothing$ used a calculation by U. Baur et al., Phys. Rev. D 56, 140 (1997). 